\title{
Cap-assisted ERCP with a forward-viewing gastroscope as a rescue endoscopic intervention in patients with Billroth II anatomy
}

\author{
Constantinos P. Anastassiades - Wajeeh Salah • \\ Eric M. Pauli · Jeffrey M. Marks · Amitabh Chak
}

Received: 9 August 2012/ Accepted: 6 January 2013/Published online: 8 February 2013

(C) Springer Science+Business Media New York 2013

\begin{abstract}
Background ERCP, especially therapeutic, is difficult in patients with Billroth II surgical reconstruction and is associated with a higher rate of complications. This has led to controversy on the choice between a forward-viewing and side-viewing endoscope for performing the procedure. A previous case series from Asia reported a high rate of success with a cap-fitted ERCP technique. To our knowledge, the utility of cap-assisted ERCP with a forward-viewing gastroscope when other techniques fail has not been reported. We describe and demonstrate a novel rescue approach using a cap-fitted, forward-viewing gastroscope in patients with Billroth II anatomy, when attempts with duodenoscopes, pediatric colonoscopes, and gastroscopes previously failed. Methods Retrospective case series. Inclusion criteria were: (a) documented Billroth II anatomy; and (b) use of cap-assisted ERCP as a rescue intervention on the first endoscopic encounter after failed attempts to perform ERCP with a duodenoscope. Patients were excluded if they successfully underwent ERCP with a duodenoscope. One advanced endoscopist and one advanced endoscopy fellow performed all but one of the procedures.
\end{abstract}

Electronic supplementary material The online version of this article (doi:10.1007/s00464-013-2814-x) contains supplementary material, which is available to authorized users.

C. P. Anastassiades $(\bowtie) \cdot$ W. Salah $\cdot$ A. Chak

Division of Gastroenterology, Department of Medicine,

Louis Stokes Cleveland VA Medical Center, University

Hospitals Case Medical Center and Case Western Reserve

University, Cleveland, OH, USA

e-mail: constantinos.anastassiades@uhhospitals.org

E. M. Pauli · J. M. Marks

Department of Surgery, University Hospitals Case Medical Center and Case Western Reserve University, Cleveland, $\mathrm{OH}$, USA
Results Five cap-assisted ERCP procedures were performed in three patients with Billroth II anatomy. A wide variety of diagnostic and therapeutic endoscopic maneuvers were technically feasible and successful, including the endoscopic treatment of an afferent limb perforation caused by a duodenoscope.

Conclusions Cap-assisted ERCP is a novel and underutilized technique that adds to the armamentarium of experienced therapeutic endoscopists. This approach may help ensure a successful endoscopic outcome and spare patients with Billroth II anatomy a percutaneous or surgical approach when ERCP with a duodenoscope, pediatric colonoscope or non-cap-fitted gastroscope fails.

Keywords Cap-assisted ERCP · Billroth II anatomy · Forward-viewing gastroscope

Disclosures Dr. Jeffrey M. Marks is a consultant for Olympus, WL Gore, and Covidien and is an advisory board member of Apollo Endosurgery. Dr. Amitabh Chak is a consultant for U.S. Endoscopy and an advisory board member of Xlumena. He provides research support for Olympus America. Drs. Constantinos P. Anastassiades, Wajeeh Salah, and Eric M. Pauli have no conflict of interest or financial ties to disclose. 